\title{
Solution of Fractional Partial Differential Equations in Fluid Mechanics by Extension of Some Iterative Method
}

\author{
A. A. Hemeda \\ Department of Mathematics, Faculty of Science, Tanta University, Tanta 31527, Egypt \\ Correspondence should be addressed to A. A. Hemeda; aahemeda@yahoo.com
}

Received 23 September 2013; Accepted 7 November 2013

Academic Editor: Tie-cheng Xia

Copyright ( 2013 A. A. Hemeda. This is an open access article distributed under the Creative Commons Attribution License, which permits unrestricted use, distribution, and reproduction in any medium, provided the original work is properly cited.

\begin{abstract}
An extension of the so-called new iterative method (NIM) has been used to handle linear and nonlinear fractional partial differential equations. The main property of the method lies in its flexibility and ability to solve nonlinear equations accurately and conveniently. Therefore, a general framework of the NIM is presented for analytical treatment of fractional partial differential equations in fluid mechanics. The fractional derivatives are described in the Caputo sense. Numerical illustrations that include the fractional wave equation, fractional Burgers equation, fractional KdV equation, fractional Klein-Gordon equation, and fractional Boussinesq-like equation are investigated to show the pertinent features of the technique. Comparison of the results obtained by the NIM with those obtained by both Adomian decomposition method (ADM) and the variational iteration method (VIM) reveals that the NIM is very effective and convenient. The basic idea described in this paper is expected to be further employed to solve other similar linear and nonlinear problems in fractional calculus.
\end{abstract}

\section{Introduction}

Recent advances of fractional differential equations are stimulated by new examples of applications in fluid mechanics, viscoelasticity, mathematical biology, electrochemistry, and physics. For example, the nonlinear oscillation of earthquake can be modeled with fractional derivatives [1], and the fluiddynamic traffic model with fractional derivatives [2] can eliminate the deficiency arising from the assumption of continuum traffic flow. Based on experimental data fractional partial differential equations for seepage flow in porous media are suggested in [3], and differential equations with fractional order have recently proved to be valuable tools to the modeling of many physical phenomena [4]. Different fractional partial differential equations have been studied and solved including the space-time-fractional diffusion-wave equation [5-7], the fractional advection-dispersion equation $[8,9]$, the fractional telegraph equation [10], the fractional KdV equation [11], and the linear inhomogeneous fractional partial differential equations [12].

The NIM [13-15] is a suitable approach to provide analytical approximation to linear and nonlinear problems and it is particularly valuable as tool for scientists and applied mathematicians, because it provides immediate and visible symbolic terms of analytical solutions, as well as numerical approximate solutions to both linear and nonlinear differential equations without linearization or discretization. The NIM, proposed by Daftardar-Gejji and Jafari in 2006 [13] and improved by Hemeda [14], was successfully applied to a variety of linear and nonlinear equations such as algebraic equations, integral equations, integrodifferential equations, ordinary and partial differential equations of integer and fractional order, and systems of equations as well. NIM is simple to understand and easy to implement using computer packages and yields better results [15] than the existing ADM [16], homotopy perturbation method (HPM) [17], or VIM [18].

The objective of this work is to extend the application of the NIM to obtain analytical solutions to some fractional partial differential equations in fluid mechanics. These equations include wave equation, Burgers equation, $\mathrm{KdV}$ equation, Klein-Gordon equation, and Boussinesq-like equation. The NIM is a computational method that yields analytical solutions and has certain advantages over standard numerical methods. It is free from rounding-off errors as it does not involve discretization and does not require large computer 
obtained memory or power. The method introduces the solution in the form of a convergent fractional series with elegantly computable terms. The corresponding solutions of the integer order equations are found to follow as special cases of those of fractional order equations. The obtained results of the NIM are compared with those obtained by both ADM [19, 20] and the VIM [21-25] which confirm that this method is very effective and convenient to these equations and to other similar equations where it has the advantage that there is no need to calculate Adomian's polynomials for the nonlinear problems as in the ADM. For more details see [26-31].

Throughout this work, fractional partial differential equations are obtained from the corresponding integer order equations by replacing the first-order or the second-order time derivative by a fractional in the Caputo sense [32] of order $\alpha$ with $0<\alpha \leq 1$ or $1<\alpha \leq 2$.

\section{Preliminaries and Notations}

In this section we give some basic definitions and properties of the fractional calculus theory which are used further in this work.

Definition 1. A real function $f(t), t>0$, is said to be in the space $C_{\mu}, \mu \in R$ if there exists a real number $p(>\mu)$, such that $f(t)=t^{p} f_{1}(t)$, where $f_{1}(t) \in C[0, \infty)$, and it is said to be in the space $C_{\mu}^{m}$ if and only if $f^{(m)} \in C_{\mu}, m \in N$.

Definition 2. The Riemann-Liouville fractional integral operator of order $\alpha \geq 0$ of a function $f \in C_{\mu}, \mu \geq-1$, is defined as

$$
\begin{gathered}
I^{\alpha} f(t)=\frac{1}{\Gamma(\alpha)} \int_{0}^{t}(t-\tau)^{\alpha-1} f(\tau) d \tau, \quad \alpha>0, t>0, \\
I^{0} f(t)=f(t) .
\end{gathered}
$$

Properties of the operators $I^{\alpha}$ can be found in [32-35]; we mention only the following: for $f \in C_{\mu}, \mu \geq-1, \alpha, \beta \geq 0$, and $v>-1$,

$$
\begin{aligned}
& \text { (1) } I^{\alpha} I^{\beta} f(t)=I^{\alpha+\beta} f(t), \\
& \text { (2) } I^{\alpha} I^{\beta} f(t)=I^{\beta} I^{\alpha} f(t), \\
& \text { (3) } I^{\alpha} t^{v}=(\Gamma(v+1) / \Gamma(v+1+\alpha)) t^{\alpha+\nu} .
\end{aligned}
$$

The Riemann-Liouville derivative has certain disadvantages when trying to model real-world phenomena with fractional differential equations. Therefore, we will introduce a modified fractional differential operator $D^{\alpha}$ proposed by Caputo in this work on the theory of viscoelasticity [32].

Definition 3. The fractional derivative of $f(t)$ in the Caputo sense is defined as

$$
\begin{array}{r}
D^{\alpha} f(t)=I^{m-\alpha} D^{m} f(t)=\frac{1}{\Gamma(m-\alpha)} \int_{0}^{t}(t-\tau)^{m-\alpha-1} f(\tau) d \tau, \\
\text { for } m-1<\alpha \leq m, m \in N, t>0, f \in C_{-1}^{m} .
\end{array}
$$

Also, we need here two of its basic properties.
Lemma 4. If $m-1<\alpha \leq m, m \in N$, and $f \in C_{\mu}^{m}, \mu \geq-1$, then

$$
\begin{gathered}
D^{\alpha} I^{\alpha} f(t)=f(t), \\
I^{\alpha} D^{\alpha} f(t)=f(t)-\sum_{k=0}^{m-1} f^{(k)}\left(0^{+}\right) \frac{t^{k}}{k !}, \quad t>0 .
\end{gathered}
$$

The Caputo fractional derivative is considered here because it allows traditional initial and boundary conditions to be included in the formulation of the problem [36]. In this work, we consider the one-dimensional linear inhomogeneous fractional partial differential equations in fluid mechanics, where the unknown function $u(x, t)$ is assumed to be a causal function of time, that is, vanishing for $t<0$. The fractional derivative is taken in Caputo sense as follows.

Definition 5. For $m$ to be the smallest integer that exceeds $\alpha$, the Caputo time-fractional derivative operator of order $\alpha>0$ is defined as

$$
\begin{aligned}
D_{t}^{\alpha} u(x, t) & =\frac{\partial^{\alpha} u}{\partial t^{\alpha}} \\
& = \begin{cases}\frac{1}{\Gamma(m-\alpha)} \int_{0}^{t}(t-\tau)^{m-\alpha-1} \frac{\partial^{m}}{\partial \tau^{m}} u(x, \tau) d \tau, \\
m-1<\alpha \leq m, \\
\frac{\partial^{m}}{\partial t^{m}} u(x, t), & \alpha=m, m \in N .\end{cases}
\end{aligned}
$$

\section{New Iterative Method (NIM)}

To illustrate the basic idea of the NIM, consider the following general functional equation $[13,14,26,27]$ :

$$
u(x)=f(x)+N(u(x)),
$$

where $N$ is a nonlinear operator from a Banach space $B \rightarrow B$ and $f$ is a known function (element) of the Banach space $B$. We are looking for a solution $u$ of (5) having the series form

$$
u(x)=\sum_{i=0}^{\infty} u_{i}(x)
$$

The nonlinear operator $N$ can be decomposed as

$$
N\left(\sum_{i=0}^{\infty} u_{i}\right)=N\left(u_{0}\right)+\sum_{i=1}^{\infty}\left\{N\left(\sum_{j=0}^{i} u_{j}\right)-N\left(\sum_{j=0}^{i-1} u_{j}\right)\right\} \text {. }
$$

From (6) and (7), (5) is equivalent to

$$
\sum_{i=0}^{\infty} u_{i}=f+N\left(u_{0}\right)+\sum_{i=1}^{\infty}\left\{N\left(\sum_{j=0}^{i} u_{j}\right)-N\left(\sum_{j=0}^{i-1} u_{j}\right)\right\} \text {. }
$$

The required solution for (5) can be obtained recurrently from the recurrence relation:

$$
u_{0}=f, \quad u_{1}=N\left(u_{0}\right),
$$

$$
\begin{aligned}
u_{n+1}= & N\left(u_{0}+u_{1}+\cdots+u_{n}\right) \\
& -N\left(u_{0}+u_{1}+\cdots+u_{n-1}\right), \quad n=1,2, \ldots
\end{aligned}
$$


Then

$$
\begin{gathered}
\left(u_{1}+u_{2}+\cdots+u_{n+1}\right) \\
=N\left(u_{0}+u_{1}+\cdots+u_{n}\right), \quad n=1,2, \ldots, \\
u=f+\sum_{i=0}^{\infty} u_{i} .
\end{gathered}
$$

The $n$-term approximate solution of (5) and (6) is given by

$$
u(x)=u_{0}+u_{1}+\cdots+u_{n-1} .
$$

Remark 6. If $N$ is a contraction, that is, $\|N(x)-N(y)\| \leq$ $k\|x-y\|, 0<k<1$, then

$$
\left\|u_{n+1}\right\| \leq k^{n+1}\left\|u_{0}\right\|, \quad n=0,1,2, \ldots
$$

Proof. From (9), we have

$$
\begin{gathered}
u_{0}=f, \quad\left\|u_{1}\right\|=\left\|N\left(u_{0}\right)\right\| \leq k\left\|u_{0}\right\|, \\
\left\|u_{2}\right\|=\left\|N\left(u_{0}+u_{1}\right)-N\left(u_{0}\right)\right\| \leq k\left\|u_{1}\right\| \leq k^{2}\left\|u_{0}\right\|, \\
\left\|u_{3}\right\|=\left\|N\left(u_{0}+u_{1}+u_{2}\right)-N\left(u_{0}+u_{1}\right)\right\| \\
\leq k\left\|u_{2}\right\| \leq k^{3}\left\|u_{0}\right\|, \\
\vdots \\
\left\|u_{n+1}\right\|=\left\|N\left(u_{0}+\cdots+u_{n}\right)-N\left(u_{0}+\cdots+u_{n-1}\right)\right\| \\
\leq k\left\|u_{n}\right\| \leq k^{n+1}\left\|u_{0}\right\|, \quad n=0,1,2, \ldots,
\end{gathered}
$$

and the series $\sum_{i=0}^{\infty} u_{i}$ absolutely and uniformly converges to a solution of (5) [37], which is unique in view of the Banach fixed point theorem [38]. For more details about NIM see [39].

3.1. Reliable Algorithm. To illustrate the basic idea of the reliable algorithm, we consider the general fractional partial differential equation of arbitrary fractional order:

$$
\frac{\partial^{\alpha} u(x, t)}{\partial t^{\alpha}}=A(u, \partial u)+B(x, t), \quad m-1<\alpha \leq m,
$$

with the initial conditions

$$
\frac{\partial^{k} u(x, 0)}{\partial t^{k}}=h_{k}(x), \quad k=0,1,2, \ldots, m-1, m \in N,
$$

where $A$ is a nonlinear function of $u, \partial u$ (partial derivatives of $u$ with respect to $x$ and $t$ ) and $B$ is the source function. In view of the fractional calculus and the properties of the fractional integral operators, the initial value problem (14a) and (14b) is equivalent to the following fractional integral equation:

$$
u(x, t)=\sum_{k=0}^{m-1} h_{k}(x) \frac{t^{k}}{k !}+I_{t}^{\alpha} B+I_{t}^{\alpha} A=f+N(u),
$$

where $f=\sum_{k=0}^{m-1} h_{k}(x)\left(t^{k} / k !\right)+I_{t}^{\alpha} B$ and $N(u)=I_{t}^{\alpha} A$. We get the solution of (15) by employing the recurrence relation (9).
Remark 7. When the general functional equation (5) is linear, the recurrence relation (9) can be simplified in the form

$$
\begin{gathered}
u_{0}=f, \\
u_{n+1}=N\left(u_{n}\right), \quad n=0,1,2, \ldots .
\end{gathered}
$$

Proof. From the properties of integration, in case $N$ is an integral operator, we have

$$
\begin{aligned}
u_{n+1}= & N\left(u_{0}+\cdots+u_{n-1}+u_{n}\right)-N\left(u_{0}+\cdots+u_{n-1}\right) \\
= & I_{x}^{\alpha}\left[u_{0}+\cdots+u_{n-1}+u_{n}\right]-I_{x}^{\alpha}\left[u_{0}+\cdots+u_{n-1}\right] \\
= & I_{x}^{\alpha}\left[u_{0}\right]+\cdots+I_{x}^{\alpha}\left[u_{n-1}\right]+I_{x}^{\alpha}\left[u_{n}\right] \\
& -I_{x}^{\alpha}\left[u_{0}\right]-\cdots-I_{x}^{\alpha}\left[u_{n-1}\right] \\
= & I_{x}^{\alpha}\left[u_{n}\right]=N\left(u_{n}\right), \quad n=0,1,2, \ldots
\end{aligned}
$$

In case $N$ is a differential operator, we obtain the same result.

3.2. Convergence of NIM. Now we analyze the convergence of the NIM for solving any general functional equation (5). Let $e=u^{*}-u$, where $u^{*}$ is the exact solution, $u$ is the approximate solution, and $e$ is the error in the solution of (5); obviously $e$ satisfies (5); that is,

$$
e(x)=f(x)+N(e(x))
$$

and the recurrence relation (9) becomes

$$
\begin{gathered}
e_{0}=f, \quad e_{1}=N\left(e_{0}\right), \\
e_{n+1}=N\left(e_{0}+e_{1}+\cdots+e_{n}\right) \\
-N\left(e_{0}+e_{1}+\cdots+e_{n-1}\right), \quad n=1,2, \ldots
\end{gathered}
$$

If $\|N(x)-N(y)\| \leq k\|x-y\|, 0<k<1$, then

$$
\begin{gathered}
e_{0}=f, \quad\left\|e_{1}\right\|=\left\|N\left(e_{0}\right)\right\| \leq k\left\|e_{0}\right\|, \\
\left\|e_{2}\right\|=\left\|N\left(e_{0}+e_{1}\right)-N\left(e_{0}\right)\right\| \\
\leq k\left\|e_{1}\right\| \leq k^{2}\left\|e_{0}\right\|, \\
\left\|e_{3}\right\|=\left\|N\left(e_{0}+e_{1}+e_{2}\right)-N\left(e_{0}+e_{1}\right)\right\| \\
\leq k\left\|e_{2}\right\| \leq k^{3}\left\|e_{0}\right\|, \\
\quad \vdots \\
\left\|e_{n+1}\right\|=\left\|N\left(e_{0}+\cdots+e_{n}\right)-N\left(e_{0}+\cdots+e_{n-1}\right)\right\| \\
\leq k\left\|e_{n}\right\| \leq k^{n+1}\left\|e_{0}\right\|, \quad n=0,1,2, \ldots
\end{gathered}
$$

Thus $e_{n+1} \rightarrow 0$ as $n \rightarrow \infty$, which proves the convergence of the NIM for solving the general functional equation (5).

Remark 8. For linear problems, we get the solution of (5) by employing the recurrence relation (16) in place of the recurrence relation (9). 


\section{Numerical Examples}

4.1. Linear Problems. To incorporate the above discussion, three linear fractional partial differential equations will be studied. The NIM is used to obtain the exact solution of these problems.

Example 1. Consider the following one-dimensional linear inhomogeneous fractional wave equation:

$$
\begin{array}{r}
D_{x}^{\alpha} u+u_{x}=\frac{t^{1+\alpha}}{\Gamma(2-\alpha)} \sin x+t \cos x, \\
t>0, x \in R, 0<\alpha \leq 1,
\end{array}
$$

subject to the initial condition

$$
u(x, 0)=0 \text {. }
$$

Problem (21a) and (21b) is solved in [40] by using the ADM; the first few components of solution are as follows:

$$
\begin{gathered}
u_{0}(x, t)=t \sin x+\frac{t^{1+\alpha}}{\Gamma(2+\alpha)} \cos x, \\
u_{1}(x, t)=-\frac{t^{1+\alpha}}{\Gamma(2+\alpha)} \cos x+\frac{t^{1+2 \alpha}}{\Gamma(2+2 \alpha)} \sin x, \\
u_{2}(x, t)=-\frac{t^{1+2 \alpha}}{\Gamma(2+2 \alpha)} \sin x-\frac{t^{1+3 \alpha}}{\Gamma(2+3 \alpha)} \cos x,
\end{gathered}
$$

The solution for (21a) and (21b) in series form is given by

$$
\begin{aligned}
u(x, t)= & t \sin x+\frac{t^{1+\alpha}}{\Gamma(2+\alpha)} \cos x-\frac{t^{1+\alpha}}{\Gamma(2+\alpha)} \cos x \\
& +\frac{t^{1+2 \alpha}}{\Gamma(2+2 \alpha)} \sin x-\frac{t^{1+2 \alpha}}{\Gamma(2+2 \alpha)} \sin x \\
& -\frac{t^{1+3 \alpha}}{\Gamma(2+3 \alpha)} \cos x+\cdots
\end{aligned}
$$

Canceling the noise terms and keeping the nonnoise terms in (23) yield the exact solution of (21a) and (21b) given by $u(x, t)=t \sin x$ which is easily verified.

Also, problem (21a) and (21b) is solved in [40] by using the VIM. By beginning with $u_{0}=0$, the following approximations can be obtained:

$$
\begin{gathered}
u_{1}(x, t)=t \sin x+\frac{t^{1+\alpha}}{\Gamma(2+\alpha)} \cos x, \\
u_{2}(x, t)=t \sin x+\frac{t^{1+\alpha}}{\Gamma(2+\alpha)} \cos x \\
-\frac{t^{1+\alpha}}{\Gamma(2+\alpha)} \cos x+\frac{t^{1+2 \alpha}}{\Gamma(2+2 \alpha)} \sin x,
\end{gathered}
$$

$$
\begin{aligned}
u_{3}(x, t)= & t \sin x+\frac{t^{1+\alpha}}{\Gamma(2+\alpha)} \cos x-\frac{t^{1+\alpha}}{\Gamma(2+\alpha)} \cos x \\
& +\frac{t^{1+2 \alpha}}{\Gamma(2+2 \alpha)} \sin x-\frac{t^{1+2 \alpha}}{\Gamma(2+2 \alpha)} \sin x \\
& -\frac{t^{1+3 \alpha}}{\Gamma(2+3 \alpha)} \cos x .
\end{aligned}
$$

As in (23), canceling the noise terms and keeping the nonnoise terms yield the exact solution of (21a) and (21b).

According to the NIM and by (15) and (16), we obtain.

$$
u_{0}(x, t)=t \sin x+\frac{t^{1+\alpha}}{\Gamma(2+\alpha)} \cos x .
$$

Therefore, the initial value problem (2la) and (21b) is equivalent to the integral equation:

$$
u(x, t)=t \sin x+\frac{t^{1+\alpha}}{\Gamma(2+\alpha)} \cos x-I_{t}^{\alpha}\left[u_{x}\right] .
$$

Let $N(u)=-I_{t}^{\alpha}\left[u_{x}\right]$; we can obtain the following first few components of the new iterative solution for (21a) and (21b):

$$
\begin{gathered}
u_{0}(x, t)=t \sin x+\frac{t^{1+\alpha}}{\Gamma(2+\alpha)} \cos x, \\
u_{1}(x, t)=-\frac{t^{1+\alpha}}{\Gamma(2+\alpha)} \cos x+\frac{t^{1+2 \alpha}}{\Gamma(2+2 \alpha)} \sin x, \\
u_{2}(x, t)=-\frac{t^{1+2 \alpha}}{\Gamma(2+2 \alpha)} \sin x-\frac{t^{1+3 \alpha}}{\Gamma(2+3 \alpha)} \cos x,
\end{gathered}
$$

The solution in series form is given by

$$
\begin{aligned}
u(x, t)= & t \sin x+\frac{t^{1+\alpha}}{\Gamma(2+\alpha)} \cos x \\
& -\frac{t^{1+\alpha}}{\Gamma(2+\alpha)} \cos x+\frac{t^{1+2 \alpha}}{\Gamma(2+2 \alpha)} \sin x \\
& -\frac{t^{1+2 \alpha}}{\Gamma(2+2 \alpha)} \sin x-\frac{t^{1+3 \alpha}}{\Gamma(2+3 \alpha)} \cos x+\cdots
\end{aligned}
$$

Canceling the noise terms and keeping the non-noise terms in (28) yield the exact solution of (21a) and (21b).

From (23), (24), and (28), it is clear that the three methods are the same in solving (21a) and (21b).

Example 2. Consider the following one-dimensional linear inhomogeneous fractional Burgers equation

$$
\begin{aligned}
D_{t}^{\alpha} & u+u_{x}-u_{x x} \\
& =\frac{2 t^{2-\alpha}}{\Gamma(3-\alpha)}+2 x-2, \quad t>0, x \in R, 0<\alpha \leq 1,
\end{aligned}
$$

subject to the initial condition

$$
u(x, 0)=x^{2} .
$$


Problem (29a) and (29b) is solved in [40] by using the ADM; the first few components of solution are as follows:

$$
\begin{gathered}
u_{0}(x, t)=x^{2}+t^{2}+\frac{t^{\alpha}}{\Gamma(1+\alpha)}(2 x-2), \\
u_{1}(x, t)=-\frac{t^{\alpha}}{\Gamma(1+\alpha)}(2 x-2)-\frac{2 t^{2 \alpha}}{\Gamma(1+2 \alpha)}, \\
u_{2}(x, t)=\frac{2 t^{2 \alpha}}{\Gamma(1+2 \alpha)}, \quad u_{3}(x, t)=0 .
\end{gathered}
$$

Therefore, the exact solution is given by $u(x, t)=x^{2}+t^{2}$.

Also, problem (29a) and (29b) is solved in [40] by using the VIM with $u_{0}=x^{2}$; the following approximations can be obtained:

$$
u_{0}(x, t)=\cdots=u_{n}(x, t)=x^{2}+t^{2} .
$$

The exact solution $u(x, t)=x^{2}+t^{2}$ follows immediately.

According to the NIM, by (15) and (16), we obtain

$$
u_{0}(x, t)=x^{2}+t^{2}+\frac{t^{\alpha}}{\Gamma(1+\alpha)}(2 x-2) \text {. }
$$

Therefore, the initial value problem (29a) and (29b) is equivalent to the integral equation:

$$
u(x, t)=x^{2}+t^{2}+\frac{t^{\alpha}}{\Gamma(1+\alpha)}(2 x-2)-I_{t}^{\alpha}\left[u_{x}-u_{x x}\right]
$$

Let $N(u)=-I_{t}^{\alpha}\left[u_{x}-u_{x x}\right]$; we obtain the following first few components of the new iterative solution for (29a) and (29b):

$$
\begin{gathered}
u_{0}(x, t)=x^{2}+t^{2}+\frac{t^{\alpha}}{\Gamma(1+\alpha)}(2 x-2), \\
u_{1}(x, t)=-\frac{t^{\alpha}}{\Gamma(1+\alpha)}(2 x-2)-\frac{2 t^{2 \alpha}}{\Gamma(1+2 \alpha)}, \\
u_{2}(x, t)=\frac{2 t^{2 \alpha}}{\Gamma(1+2 \alpha)}, \quad u_{3}(x, t)=0 .
\end{gathered}
$$

Therefore, the exact solution $u(x, t)=x^{2}+t^{2}$ follows immediately.

Example 3. Consider the following one-dimensional linear inhomogeneous fractional Klein-Gordon equation:

$$
\begin{aligned}
D_{t}^{\alpha} u & -u_{x x}+u \\
& =6 x^{3} t+\left(x^{3}-6 x\right) t^{3}, \quad t>0, x \in R, 1<\alpha \leq 2,
\end{aligned}
$$

subject to the initial conditions

$$
u(x, 0)=0, \quad u_{t}(x, 0)=0 .
$$

In view of the ADM [40], the first few components of solution for (35a) and (35b) are derived as follows:

$$
\begin{aligned}
u_{0}(x, t) & =6 x^{3} \frac{t^{1+\alpha}}{\Gamma(2+\alpha)}+6\left(x^{3}-6 x\right) \frac{t^{3+\alpha}}{\Gamma(4+\alpha)}, \\
u_{1}(x, t)= & 36 x \frac{t^{1+2 \alpha}}{\Gamma(2+2 \alpha)}+36 x \frac{t^{3+2 \alpha}}{\Gamma(4+2 \alpha)} \\
& -6 x^{3} \frac{t^{1+2 \alpha}}{\Gamma(2+2 \alpha)}-6\left(x^{3}-6 x\right) \frac{t^{3+2 \alpha}}{\Gamma(4+2 \alpha)},
\end{aligned}
$$

The solution in series form is given by

$$
\begin{aligned}
u(x, t)= & 6 x^{3} \frac{t^{1+\alpha}}{\Gamma(2+\alpha)}+6\left(x^{3}-6 x\right) \frac{t^{3+\alpha}}{\Gamma(4+\alpha)} \\
& +36 x \frac{t^{1+2 \alpha}}{\Gamma(2+2 \alpha)}+36 x \frac{t^{3+2 \alpha}}{\Gamma(4+2 \alpha)} \\
& -6 x^{3} \frac{t^{1+2 \alpha}}{\Gamma(2+2 \alpha)}-6\left(x^{3}-6 x\right) \frac{t^{3+2 \alpha}}{\Gamma(4+2 \alpha)}+\cdots
\end{aligned}
$$

In view of the VIM [40], with $u_{0}=0$, the following approximations for (35a) and (35b) are obtained:

$$
\begin{aligned}
u_{1}(x, t) & =(\alpha-1)\left[6 x^{3} \frac{t^{1+\alpha}}{\Gamma(2+\alpha)}+6\left(x^{3}-6 x\right) \frac{t^{3+\alpha}}{\Gamma(4+\alpha)}\right] \\
u_{2}(x, t) & \\
= & 6 x^{3} \frac{t^{1+\alpha}}{\Gamma(2+\alpha)}+6\left(x^{3}-6 x\right) \frac{t^{3+\alpha}}{\Gamma(4+\alpha)}-(\alpha-1)^{2} \\
& \times\left[6\left(x^{3}-6 x\right) \frac{t^{1+\alpha}}{\Gamma(2+\alpha)}+6\left(x^{3}-6 x\right) \frac{t^{3+\alpha}}{\Gamma(4+\alpha)}\right] \\
& +\cdots
\end{aligned}
$$

According to the NIM, by (15) and (16), we can obtain

$$
u_{0}(x, t)=6 x^{3} \frac{t^{1+\alpha}}{\Gamma(2+\alpha)}+6\left(x^{3}-6 x\right) \frac{t^{3+\alpha}}{\Gamma(4+\alpha)} .
$$

Therefore, the initial value problem (35a) and (35b) is equivalent to the integral equation:

$$
\begin{aligned}
u(x, t)= & 6 x^{3} \frac{t^{1+\alpha}}{\Gamma(2+\alpha)}+6\left(x^{3}-6 x\right) \frac{t^{3+\alpha}}{\Gamma(4+\alpha)} \\
& +I_{t}^{\alpha}\left[u_{x x}-u\right] .
\end{aligned}
$$


Let $N(u)=I_{t}^{\alpha}\left[u_{x x}-u\right]$; we can obtain the following first few components of the new iterative solution for (35a) and (35b):

$$
\begin{aligned}
u_{0}(x, t) & =6 x^{3} \frac{t^{1+\alpha}}{\Gamma(2+\alpha)}+6\left(x^{3}-6 x\right) \frac{t^{3+\alpha}}{\Gamma(4+\alpha)}, \\
u_{1}(x, t)= & 36 x \frac{t^{1+2 \alpha}}{\Gamma(2+2 \alpha)}+36 x \frac{t^{3+2 \alpha}}{\Gamma(4+2 \alpha)} \\
& -6 x^{3} \frac{t^{1+2 \alpha}}{\Gamma(2+2 \alpha)}-6\left(x^{3}-6 x\right) \frac{t^{3+2 \alpha}}{\Gamma(4+2 \alpha)},
\end{aligned}
$$

The solution for (35a) and (35b) in series form is given by

$$
\begin{aligned}
u(x, t)= & 6 x^{3} \frac{t^{1+\alpha}}{\Gamma(2+\alpha)}+6\left(x^{3}-6 x\right) \frac{t^{3+\alpha}}{\Gamma(4+\alpha)} \\
& +36 x \frac{t^{1+2 \alpha}}{\Gamma(2+2 \alpha)}+36 x \frac{t^{3+2 \alpha}}{\Gamma(4+2 \alpha)} \\
& -6 x^{3} \frac{t^{1+2 \alpha}}{\Gamma(2+2 \alpha)}-6\left(x^{3}-6 x\right) \frac{t^{3+2 \alpha}}{\Gamma(4+2 \alpha)}+\cdots
\end{aligned}
$$

From (37), (38), and (42), the ADM, the VIM, and the NIM give the same solution for the classical Klein-Gordon equation (35a) and (35b) in the case $\alpha=2$ which is given by

$$
\begin{aligned}
u(x, t)= & x^{3} t^{3}+\left(x^{3}-6 x\right) \frac{6 t^{5}}{\Gamma(6)} \\
& +36 x \frac{t^{5}}{\Gamma(6)}-36 x \frac{6 t^{7}}{\Gamma(8)}-6 x^{3} \frac{t^{5}}{\Gamma(6)} \\
& -\left(x^{3}-6 x\right) \frac{6 t^{7}}{\Gamma(8)}+\cdots .
\end{aligned}
$$

Canceling the noise terms and keeping the non-noise terms in (43) yield the exact solution of (35a) and (35b) for the special case $\alpha=2$, which is given by $u(x, t)=x^{3} t^{3}$, which is easily verified.

4.2. Nonlinear Problems. For nonlinear equations in general, there exists no method that yields the exact solution and therefore only approximate solutions can be derived. In this subsection, we use the NIM to provide approximate solutions for two kinds of nonlinear time-fractional partial differential equations.

Example 4. Consider the following one-dimensional nonlinear homogeneous time-fractional KdV equation:

$$
D_{t}^{\alpha} u+6 u u_{x}+u_{x x x}=0, \quad t>0, x \in R, 0<\alpha \leq 1,
$$

subject to the initial condition

$$
u(x, 0)=\frac{1}{2} \operatorname{sech}^{2}\left(\frac{x}{2}\right) .
$$

The time-fractional $\mathrm{KdV}$ equation (44a) and (44b) is solved in $[11,40]$ by using the ADM. The solution in series form is found as

$$
\begin{aligned}
u(x, t)= & f_{0}(x)+f_{1}(x) \frac{t^{\alpha}}{\Gamma(1+\alpha)} \\
& +f_{2}(x) \frac{t^{2 \alpha}}{\Gamma(1+2 \alpha)}+f_{3}(x) \frac{t^{3 \alpha}}{\Gamma(1+3 \alpha)}+\cdots
\end{aligned}
$$

where

$$
\begin{gathered}
f_{0}(x)=\frac{1}{2} \operatorname{sech}^{2}\left(\frac{x}{2}\right), \\
f_{1}(x)=-6 f_{0} f_{0}^{\prime}-f_{0}^{\prime \prime \prime}, \\
f_{2}(x)=-6 f_{0} f_{1}^{\prime}-6 f_{1} f_{0}^{\prime}-f_{1}^{\prime \prime \prime}, \\
f_{3}(x)=-6 f_{1} f_{1}^{\prime} \frac{\Gamma(1+2 \alpha)}{\Gamma(1+\alpha)^{2}} .
\end{gathered}
$$

Also, problem (44a) and (44b) is solved in [40] by using the VIM with $u_{0}=(1 / 2) \sec h^{2}(x / 2)$; the first few approximate solutions are

$$
\begin{gathered}
u_{1}(x, t)=f_{0}(x), \\
u_{2}(x, t)=f_{0}(x)+f_{1}(x) \frac{t^{\alpha}}{\Gamma(1+\alpha)}, \\
u_{3}(x, t)=f_{0}(x)+f_{1}(x) \frac{t^{\alpha}}{\Gamma(1+\alpha)}+f_{2}(x) \frac{t^{2 \alpha}}{\Gamma(1+2 \alpha)} \\
-6 f_{1}(x) f_{1}^{\prime}(x) \frac{\Gamma(1+2 \alpha) t^{3 \alpha}}{\Gamma(1+\alpha)^{2} \Gamma(1+3 \alpha)},
\end{gathered}
$$

According to the NIM, by (15) and (9), we can obtain

$$
u_{0}(x, t)=\frac{1}{2} \operatorname{sech}^{2}\left(\frac{x}{2}\right) \text {. }
$$

Therefore, the initial value problem (44a) and (44b) is equivalent to the integral equation:

$$
u(x, t)=\frac{1}{2} \operatorname{sech}^{2}\left(\frac{x}{2}\right)-I_{t}^{\alpha}\left[6 u u_{x}+u_{x x x}\right] .
$$

Let $N(u)=-I_{t}^{\alpha}\left[6 u u_{x}+u_{x x x}\right]$; we obtain the following first few components of the new iterative solution for (44a) and (44b):

$$
\begin{gathered}
u_{0}(x, t)=f_{0}(x) \\
u_{1}(x, t)=f_{1}(x) \frac{t^{\alpha}}{\Gamma(1+\alpha)}, \\
u_{2}(x, t)=f_{2}(x) \frac{t^{2 \alpha}}{\Gamma(1+2 \alpha)}+f_{3}(x) \frac{t^{3 \alpha}}{\Gamma(1+3 \alpha)},
\end{gathered}
$$


The solution in series form is given by

$$
\begin{aligned}
u(x, t)= & f_{0}(x)+f_{1}(x) \frac{t^{\alpha}}{\Gamma(1+\alpha)} \\
& +f_{2}(x) \frac{t^{2 \alpha}}{\Gamma(1+2 \alpha)}+f_{3}(x) \frac{t^{3 \alpha}}{\Gamma(1+3 \alpha)}+\cdots
\end{aligned}
$$

where

$$
\begin{gathered}
f_{0}(x)=\frac{1}{2} \operatorname{sech}^{2}\left(\frac{x}{2}\right), \quad f_{1}(x)=-6 f_{0} f_{0}^{\prime}-f_{0}^{\prime \prime \prime}, \\
f_{2}(x)=-6 f_{0} f_{1}^{\prime}-6 f_{1} f_{0}^{\prime}-f_{1}^{\prime \prime \prime}, \\
f_{3}(x)=-6 f_{1} f_{1}^{\prime} \frac{\Gamma(1+2 \alpha)}{\Gamma(1+\alpha)^{2}} .
\end{gathered}
$$

Now, the approximate solution for (44a) and (44b) obtained by the NIM in (51) is the same solution obtained by both the ADM in (45) and the VIM in (47). Therefore, all the three methods provide the same approximate solution for the time-fractional KdV equation.

Example 5. In this example, we consider the one-dimensional nonlinear homogeneous time-fractional Boussinesq-like equation:

$$
D_{t}^{\alpha} u+\left(u^{2}\right)_{x x}-\left(u^{2}\right)_{x x x x}=0, \quad t>0, x \in R, 1<\alpha \leq 2,
$$

subject to the initial conditions

$$
u(x, 0)=\frac{4}{3} \sinh ^{2}\left(\frac{x}{4}\right), \quad u_{t}(x, 0)=-\frac{1}{3} \sinh \left(\frac{x}{2}\right) .
$$

The time-fractional Boussinesq-like equation (53a) and (53b) is solved by the ADM in [40]. The fourth-order term approximate solution in series form is given by

$$
\begin{aligned}
u(x, t)= & \frac{4}{3} \sinh ^{2}\left(\frac{x}{4}\right)-\frac{1}{3} \sinh \left(\frac{x}{2}\right) \cdot t \\
& +\frac{1}{2(3)} \cosh \left(\frac{x}{2}\right) \frac{t^{\alpha}}{\Gamma(1+\alpha)} \\
& -\frac{1}{2^{2}(3)} \sinh \left(\frac{x}{2}\right) \frac{t^{1+\alpha}}{\Gamma(2+\alpha)} \\
& +\frac{1}{2^{3}(3)} \cosh \left(\frac{x}{2}\right) \frac{t^{2 \alpha}}{\Gamma(1+2 \alpha)} \\
& -\frac{1}{2^{4}(3)} \sinh \left(\frac{x}{2}\right) \cdot \frac{t^{1+2 \alpha}}{\Gamma(2+2 \alpha)} \\
& +\frac{1}{2^{5}(3)} \cosh \left(\frac{x}{2}\right) \frac{t^{3 \alpha}}{\Gamma(1+3 \alpha)} \\
& -\frac{1}{2^{6}(3)} \sinh \left(\frac{x}{2}\right) \frac{t^{1+3 \alpha}}{\Gamma(2+3 \alpha)} .
\end{aligned}
$$

Also, problem (53a) and (53b) is solved by the VIM in [40]. The fourth-order term approximate solution, with $u_{0}=$ $(4 / 3) \sinh ^{2}(x / 4)-(1 / 3) \sinh (x / 2) \cdot t$, is given by

$$
\begin{aligned}
& u(x, t)= \frac{4}{3} \sinh ^{2}\left(\frac{x}{4}\right)-\frac{1}{3} \sinh \left(\frac{x}{2}\right) \cdot t \\
&+(\alpha-1)\left[\frac{1}{2(3)} \cosh \left(\frac{x}{2}\right) \frac{t^{\alpha}}{\Gamma(1+\alpha)}\right. \\
&\left.-\frac{1}{2^{2}(3)} \sinh \left(\frac{x}{2}\right) \frac{t^{1+\alpha}}{\Gamma(2+\alpha)}\right] \\
&+(\alpha-1)^{2} {\left[\frac{1}{2^{3}(3)} \cosh \left(\frac{x}{2}\right) \frac{t^{2 \alpha}}{\Gamma(1+2 \alpha)}\right.} \\
&+(\alpha-1)^{3} {\left[\frac{1}{2^{5}(3)} \cosh \left(\frac{x}{2}\right) \frac{t^{3 \alpha}}{\Gamma(1+3 \alpha)} \sinh \left(\frac{x}{2}\right) \frac{t^{1+2 \alpha}}{\Gamma(2+2 \alpha)}\right] } \\
&\left.-\frac{1}{2^{6}(3)} \sinh \left(\frac{x}{2}\right) \frac{t^{1+3 \alpha}}{\Gamma(2+3 \alpha)}\right] .
\end{aligned}
$$

According to the NIM, by (15) and (9), we can obtain the following first approximations:

$$
\begin{aligned}
u_{0}(x, t)= & \frac{4}{3} \sinh ^{2}\left(\frac{x}{4}\right)-\frac{1}{3} \sinh \left(\frac{x}{2}\right) \cdot t \\
u_{1}(x, t)= & \frac{1}{2(3)} \cosh \left(\frac{x}{2}\right) \frac{t^{\alpha}}{\Gamma(1+\alpha)} \\
& -\frac{1}{2^{2}(3)} \sinh \left(\frac{x}{2}\right) \frac{t^{1+\alpha}}{\Gamma(2+\alpha)}, \\
u_{2}(x, t)= & \frac{1}{2^{3}(3)} \cosh \left(\frac{x}{2}\right) \frac{t^{2 \alpha}}{\Gamma(1+2 \alpha)} \\
& -\frac{1}{2^{4}(3)} \sinh \left(\frac{x}{2}\right) \frac{t^{1+2 \alpha}}{\Gamma(2+2 \alpha)}, \\
u_{3}(x, t)= & \frac{1}{2^{5}(3)} \cosh \left(\frac{x}{2}\right) \frac{t^{3 \alpha}}{\Gamma(1+3 \alpha)} \\
& -\frac{1}{2^{6}(3)} \sinh \left(\frac{x}{2}\right) \frac{t^{1+3 \alpha}}{\Gamma(2+3 \alpha)} .
\end{aligned}
$$

The fourth-order term approximate solution in series form is given by

$$
\begin{aligned}
u(x, t)= & \frac{4}{3} \sinh ^{2}\left(\frac{x}{4}\right)-\frac{1}{3} \sinh \left(\frac{x}{2}\right) \cdot t \\
& +\frac{1}{2(3)} \cosh \left(\frac{x}{2}\right) \frac{t^{\alpha}}{\Gamma(1+\alpha)} \\
& -\frac{1}{2^{2}(3)} \sinh \left(\frac{x}{2}\right) \frac{t^{1+\alpha}}{\Gamma(2+\alpha)} \\
& +\frac{1}{2^{3}(3)} \cosh \left(\frac{x}{2}\right) \frac{t^{2 \alpha}}{\Gamma(1+2 \alpha)}
\end{aligned}
$$




$$
\begin{aligned}
& -\frac{1}{2^{4}(3)} \sinh \left(\frac{x}{2}\right) \cdot \frac{t^{1+2 \alpha}}{\Gamma(2+2 \alpha)} \\
& +\frac{1}{2^{5}(3)} \cosh \left(\frac{x}{2}\right) \frac{t^{3 \alpha}}{\Gamma(1+3 \alpha)} \\
& -\frac{1}{2^{6}(3)} \sinh \left(\frac{x}{2}\right) \frac{t^{1+3 \alpha}}{\Gamma(2+3 \alpha)},
\end{aligned}
$$

where

$$
N(u)=-I_{t}^{\alpha}\left[\left(u^{2}\right)_{x x}-\left(u^{2}\right)_{x x x x}\right] .
$$

It is interesting to point out that, for the case of $\alpha=2$, the approximate solution:

$$
\begin{aligned}
u(x, t)= & \frac{2}{3}\left[\cosh \left(\frac{x}{2}\right)\left(1+\frac{1}{2^{2}} \frac{t^{2}}{2 !}+\frac{1}{2^{4}} \frac{t^{4}}{4 !}+\cdots\right)-1\right] \\
& -\frac{2}{3} \sinh \left(\frac{x}{2}\right)\left[\frac{1}{2} t+\frac{1}{2^{3}} \frac{t^{3}}{3 !}+\frac{1}{2^{5}} \frac{t^{5}}{5 !}+\cdots\right]
\end{aligned}
$$

follows immediately upon replacing $\alpha$ by 2 in the decomposition solution (54), the variational iteration solution (55), or the new iteration solution (57), which converges to the exact solution of the Boussinesq-like equation (53a) and (53b), when $\alpha=2, u(x, t)=(4 / 3) \sinh ^{2}((x-t) / 4)$.

The advantage of the NIM in the nonlinear problems, Examples 4 and 5, is that there is no need to calculate Adomian's polynomials as done in the ADM, which means that the first method is simple and easy of the procedure of calculations over the second. Also, for more details about the solutions of the fractional differential equations by the VIM and some new asymptotic methods, you can see [41-43].

\section{Conclusion}

NIM has been known as a powerful tool for solving many functional equations such as ordinary, partial differential equations, integral equations, integrodifferential equations, and so many other equations. In this work, we have presented a general framework of the NIM for the analytical treatment of fractional partial differential equations in fluid mechanics. The present work shows the validity and great potential of the NIM for solving linear and nonlinear fractional partial differential equations. All of the examples show that the results of the NIM are in excellent agreement with those obtained by both the ADM and the VIM without calculating Adomian's polynomials for the nonlinear problems as in the ADM. The basic idea described in this work is expected to be further employed to solve other similar linear and nonlinear problems in fractional calculus.

\section{Conflict of Interests}

The author declared that there is no conflict of interests regarding the publication of this paper.

\section{References}

[1] J.H. He, "Nonlinear oscillation with fractional derivative and its applications," in Proceedings of the International Conference on Vibrating Engineering 98, Dalian, China, 1988.

[2] J. H. He, "Some applications of nonlinear fractional differential equations and their approximations," Bulletin of Science, Technology \& Society, vol. 15, no. 2, pp. 86-90, 1999.

[3] J.-H. He, "Approximate analytical solution for seepage flow with fractional derivatives in porous media," Computer Methods in Applied Mechanics and Engineering, vol. 167, no. 1-2, pp. 57-68, 1998.

[4] I. Podlubny, Fractional Differential Equations, Academic Press, New York, NY, USA, 1999.

[5] K. Al-Khaled and S. Momani, "An approximate solution for a fractional diffusion-wave equation using the decomposition method," Applied Mathematics and Computation, vol. 165, no. 2, pp. 473-483, 2005.

[6] F. Mainardi, Y. Luchko, and G. Pagnini, "The fundamental solution of the space-time fractional diffusion equation," Fractional Calculus \& Applied Analysis, vol. 4, no. 2, pp. 153-192, 2001.

[7] A. Hanyga, "Multidimensional solutions of time-fractional diffusion-wave equations," The Royal Society of London A, vol. 458, no. 2020, pp. 933-957, 2002.

[8] F. Huang and F. Liu, "The time fractional diffusion equation and the advection-dispersion equation," The ANZIAM Journal, vol. 46, no. 3, pp. 317-330, 2005.

[9] F. Huang and F. Liu, "The fundamental solution of the spacetime fractional advection-dispersion equation," Journal of Applied Mathematics \& Computing, vol. 18, no. 1-2, pp. 339-350, 2005.

[10] S. Momani, "Analytic and approximate solutions of the spaceand time-fractional telegraph equations," Applied Mathematics and Computation, vol. 170, no. 2, pp. 1126-1134, 2005.

[11] S. Momani, "An explicit and numerical solutions of the fractional KdV equation," Mathematics and Computers in Simulation, vol. 70, no. 2, pp. 110-118, 2005.

[12] L. Debnath and D. D. Bhatta, "Solutions to few linear fractional inhomogeneous partial differential equations in fluid mechanics," Fractional Calculus \& Applied Analysis, vol. 7, no. 1, pp. 2136, 2004.

[13] V. Daftardar-Gejji and H. Jafari, "An iterative method for solving nonlinear functional equations," Journal of Mathematical Analysis and Applications, vol. 316, no. 2, pp. 753-763, 2006.

[14] A. A. Hemeda, "New iterative method: application to the nthorder integro-differential equations," Information $B$, vol. 16, no. 6, pp. 3841-3852, 2013.

[15] S. Bhalekar and V. Daftardar-Gejji, "New iterative method: application to partial differential equations," Applied Mathematics and Computation, vol. 203, no. 2, pp. 778-783, 2008.

[16] G. Adomian, Solving Frontier Problems of Physics: The Decomposition Method, Kluwer, 1994.

[17] J.-H. He, "Homotopy perturbation technique," Computer Methods in Applied Mechanics and Engineering, vol. 178, no. 3-4, pp. 257-262, 1999.

[18] A. A. Hemeda, "Variational iteration method for solving nonlinear coupled equations in 2-dimensional space in fluid mechanics," International Journal of Contemporary Mathematical Sciences, vol. 7, no. 37, pp. 1839-1852, 2012.

[19] G. Adomian, "A review of the decomposition method in applied mathematics," Journal of Mathematical Analysis and Applications, vol. 135, no. 2, pp. 501-544, 1988. 
[20] T. Öziş and A. Yıldırım, "Comparison between Adomian's method and He's homotopy perturbation method," Computers \& Mathematics with Applications, vol. 56, no. 5, pp. 1216-1224, 2008.

[21] S. Momani and S. Abuasad, "Application of He's variational iteration method to Helmholtz equation," Chaos, Solitons \& Fractals, vol. 27, no. 5, pp. 1119-1123, 2006.

[22] J.-H. He, "Variational principle for nano thin film lubrication," International Journal of Nonlinear Sciences and Numerical Simulation, vol. 4, no. 3, pp. 313-314, 2003.

[23] J.-H. He, "Variational principles for some nonlinear partial differential equations with variable coefficients," Chaos, Solitons \& Fractals, vol. 19, no. 4, pp. 847-851, 2004.

[24] J.-H. He, "Variational iteration method-some recent results and new interpretations," Journal of Computational and Applied Mathematics, vol. 207, no. 1, pp. 3-17, 2007.

[25] J.-H. He and X.-H. Wu, "Variational iteration method: new development and applications," Computers \& Mathematics with Applications, vol. 54, no. 7-8, pp. 881-894, 2007.

[26] A. A. Hemeda, "Formulation and solution of nth-order derivative fuzzy integro-differential equation using new iterative method with a reliable algorithm," Journal of Applied Mathematics, vol. 2012, Article ID 325473, 17 pages, 2012.

[27] S. Bhalekar and V. Daftardar-Gejji, "Solving evolution equations using a new iterative method," Numerical Methods for Partial Differential Equations, vol. 26, no. 4, pp. 906-916, 2010.

[28] A. A. Hemeda, "Variational iteration method for solving wave equation," Computers \& Mathematics with Applications, vol. 56, no. 8, pp. 1948-1953, 2008.

[29] A. A. Hemeda, "Variational iteration method for solving nonlinear partial differential equations," Chaos, Solitons and Fractals, vol. 39, no. 3, pp. 1297-1303, 2009.

[30] A. A. Hemeda, "Homotopy perturbation method for solving systems of nonlinear coupled equations," Applied Mathematical Sciences, vol. 6, no. 93-96, pp. 4787-4800, 2012.

[31] A. A. Hemeda, "Homotopy perturbation method for solving partial differential equations of fractional order," International Journal of Mathematical Analysis, vol. 6, no. 49-52, pp. 24312448, 2012.

[32] M. Caputo, "Linear models of dissipation whose Q is almost frequency indepedent-part II," Geophysical Journal International, vol. 13, no. 5, pp. 529-539, 1967.

[33] A. Y. Luchko and R. Groreo, The Initial Value Problem for Some Fractional Differential Equations with Caputo Derivative, Preprint series A80-98, Fachbreich Mathematik und Informatik, Freic Universitat Berlin, 1998.

[34] K. S. Miller and B. Ross, An Introduction to the Fractional Calculus and Fractional Differential Equations, John Wiley \& Sons, New York, NY, USA, 1993.

[35] K. B. Oldham and J. Spanier, The Fractional Calculus, Academic Press, New York, NY, USA, 1974.

[36] I. Podlubny, "Geometric and physical interpretation of fractional integration and fractional differentiation," Fractional Calculus \& Applied Analysis, vol. 5, no. 4, pp. 367-386, 2002.

[37] Y. Cherruault, "Convergence of Adomian's method," Kybernetes, vol. 18, no. 2, pp. 31-38, 1989.

[38] A. J. Jerri, Introduction to Integral Equations with Applications, Wiley-Interscience, 2nd edition, 1999.

[39] A. A. Hemeda, "New iterative method: an application for solving fractional physical differential equations," Abstract and Applied Analysis, vol. 2013, Article ID 617010, 9 pages, 2013.
[40] Z. Odibat and S. Momani, “The variational iteration method: an efficient scheme for handling fractional partial differential equations in fluid mechanics," Computers \& Mathematics with Applications, vol. 58, no. 11-12, pp. 2199-2208, 2009.

[41] G.-C. Wu and D. Baleanu, "Variational iteration method for the Burgers' flow with fractional derivatives-new Lagrange multipliers," Applied Mathematical Modelling, vol. 37, no. 9, pp. 61836190, 2013.

[42] G. C. Wu, "New trends in the variational iteration method," Communications in Fractional Calculus, vol. 2, pp. 59-75, 2011.

[43] J.-H. He, "Asymptotic methods for solitary solutions and compactons," Abstract and Applied Analysis, vol. 2012, Article ID 916793, 130 pages, 2012. 


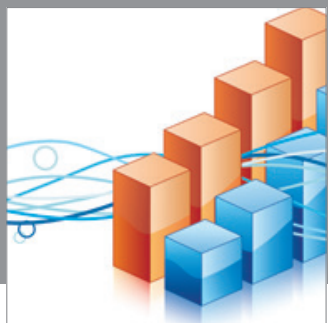

Advances in

Operations Research

mansans

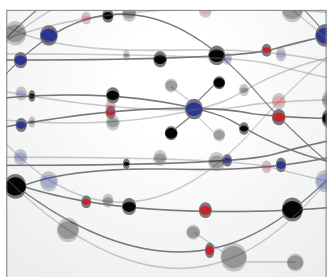

The Scientific World Journal
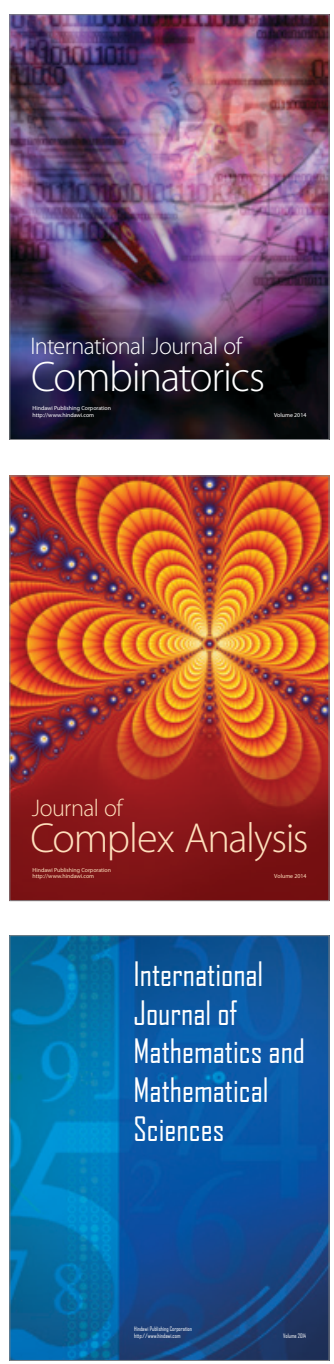
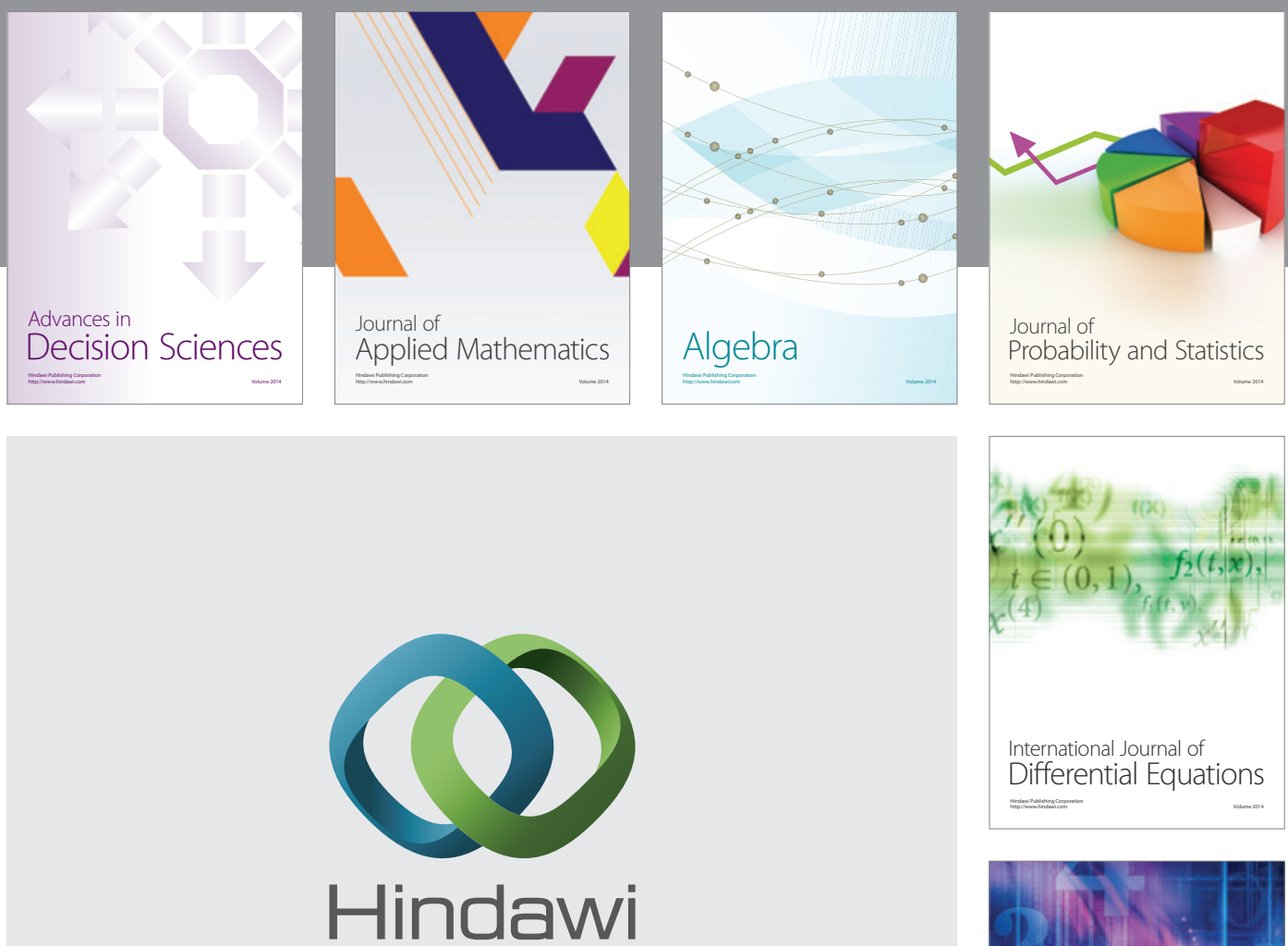

Submit your manuscripts at http://www.hindawi.com
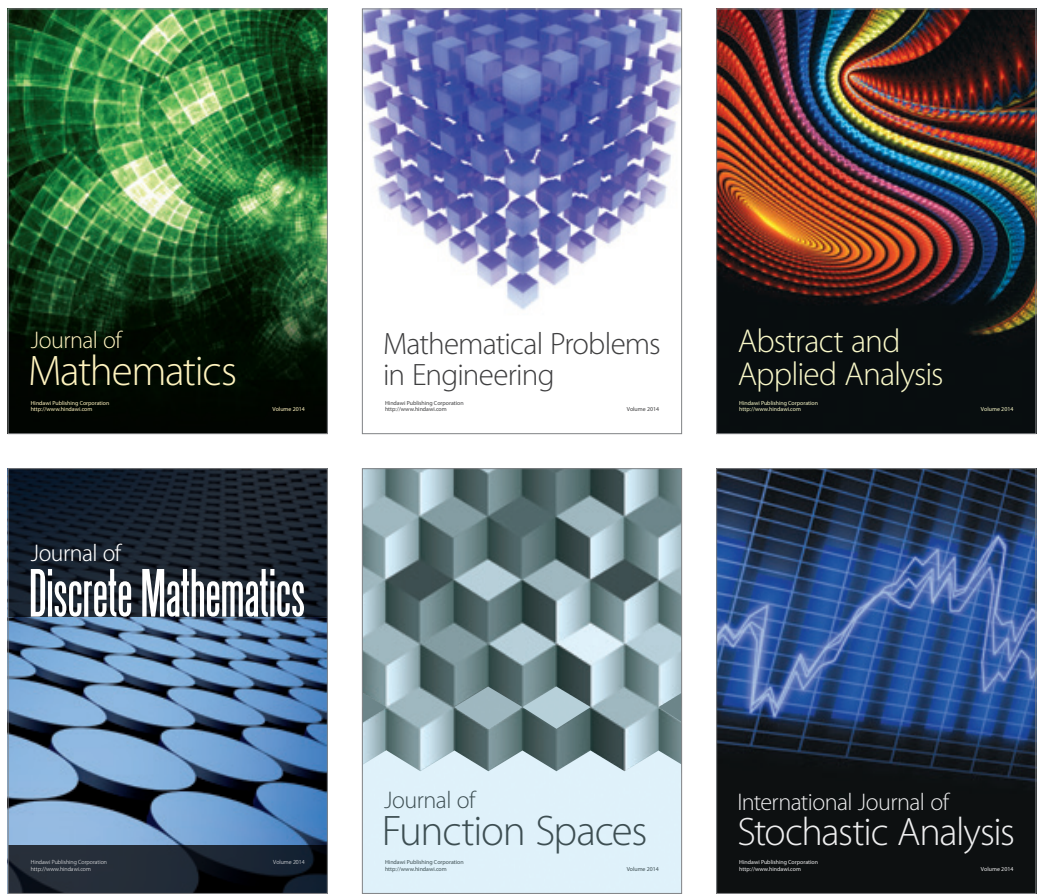

Journal of

Function Spaces

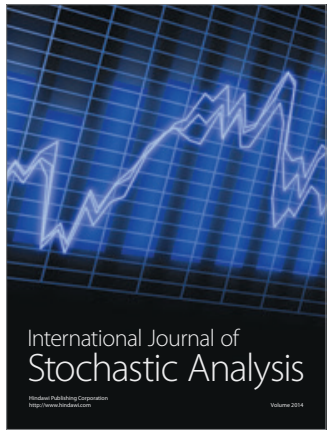

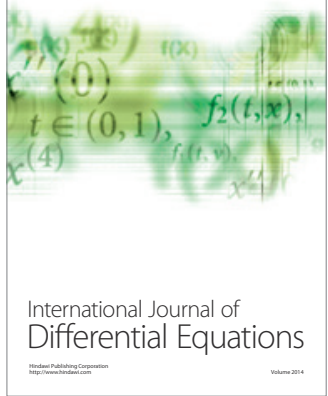
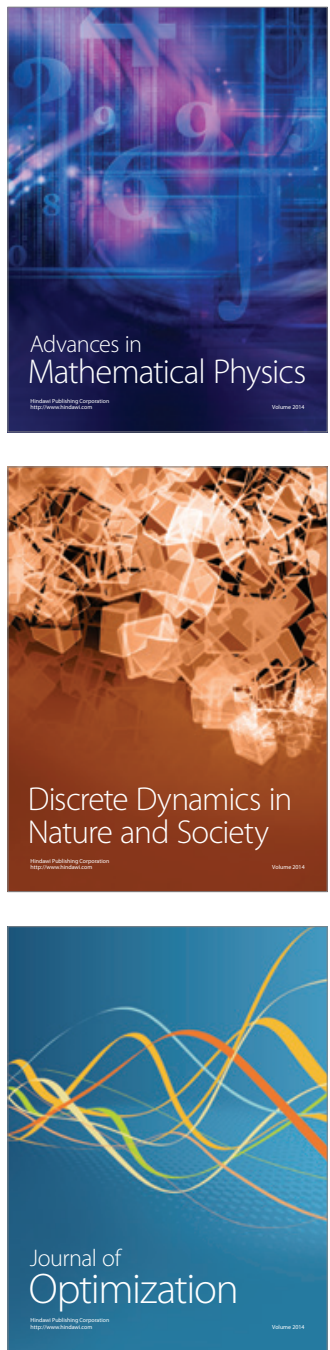